\title{
Spectroscopic characterization of human immunodeficiency virus type-1-infected plasma by principal component analysis and soft independent modeling of class analogy of visible and near-infrared spectra
}

\author{
MIRZA KHALIL BAHMANI ${ }^{1,4}$, AYYOOB KHOSRAVI ${ }^{2}$, RAMIN MIRI ${ }^{2}$, \\ YUKIE IWABU $^{3}$, KAZUYOSHI IKUTA $^{3}$ and AKIKAZU SAKUDO ${ }^{3}$ \\ ${ }^{1}$ HIV and Hepatitis Research Center, ${ }^{2}$ Medicinal and Natural Products Chemistry Research Center, \\ Shiraz University of Medical Sciences, Shiraz, Iran; ${ }^{3}$ Department of Virology, \\ Research Institute for Microbial Diseases, Osaka University, Suita, Osaka 565-0871, Japan
}

Received April 21, 2009; Accepted June 17, 2009

DOI: 10.3892/mmr_00000176

\begin{abstract}
Previous research has shown that it may be possible to diagnose infections of human immunodeficiency virus type-1 (HIV-1) using plasma by a partial least squares regression analysis of visible and near-infrared (Vis-NIR) spectra. In this study, the features of plasma in HIV-1-infected and healthy individuals were further investigated by Vis-NIR spectroscopy using principal component analysis (PCA) and soft independent modeling of class analogy (SIMCA). Although the mean Vis-NIR spectra of 33 HIV-1-infected individuals and 15 healthy donors showed only slight differences, the two groups were respectively distinguished using a score plot of the first versus second or second versus third principal components, and by a Coomans plot. The PCA loadings were generally consistent with the discriminating power of the SIMCA, indicating specific changes in Vis-NIR spectra after HIV-1-infection. The specific pattern possibly indicates $\mathrm{ROH}$ and $\mathrm{RNH}_{2}$, which may constitute specific features of components in HIV-1-infected plasma.
\end{abstract}

\section{Introduction}

Human immunodeficiency virus type-1 (HIV-1), a causative agent of acquired immunodeficiency syndrome (AIDS), can

Correspondence to: Dr Akikazu Sakudo, Department of Virology, Research Institute for Microbial Diseases, Osaka University, 3-1 Yamadaoka, Suita, Osaka 565-0871, Japan

E-mail: sakudo@biken.osaka-u.ac.jp

Present address: ${ }^{4}$ Gerash Educational Center, Shiraz University of Medical Sciences, GEC, Shohada Blv. Gerash, FARS 74419-66359, Iran

Key words: human immunodeficiency virus type-1, visible and near-infrared spectroscopy, principal component analysis, soft independent modeling of class analogy be transmitted by infected blood transfusion, sexual contact or intravenous drug use, or from mother to child (1). Infected individuals show a decreased number of CD4-positive T-cells (2). This decrease in CD4-positive T-cells appears to be a major cause of AIDS. In addition, inflammatory cellular compartments (e.g., macrophages) are major targets for HIV-1 infections (3). In the case of HIV dementia, the invasion of the central nervous system by HIV-infected and uninfected monocytic cells and the subsequent release or transport of neurotoxic proteins (Tat, gp120 and cytokines) are major pathways for neuropathogenesis (4). Although several changes in plasma after HIV-1-infection have been reported, available information is limited. From 1989, before the availability of potent anti-retroviral therapy, dyslipidemia was reported in HIV-1infected individuals (5-10). Untreated HIV-1-infected individuals show increased levels of triglycerides (11-13) and apolipoprotein B (11), decreased levels of total cholesterol $(11,12)$, high-density lipoprotein cholesterol (HDL-C) (11-13), low-density lipoprotein cholesterol (LDL-C) $(11,12)$ and preB-HDL (11), and increased levels and activities of lecithin cholesterol acyl transferase (11) and cholesteryl ester transfer protein (11). Elevations in total cholesterol and LDL-C may be related to an increased risk of atherosclerosis (14) and coronary artery disease (15) in HIV-1-infected individuals. Some HIV-1protease inhibitors cause hyperlipidemia in HIV-1-positive and HIV-1-negative individuals (16-18). Elevated cytokine levels are a feature of HIV-1-infection (19), but are also observed in other viral infections. An association between triglyceride and interferon $\alpha$ levels has been observed in individuals with AIDS $(7,20)$.

To date, several techniques, such as magnetic resonance spectroscopy (MRS) (21-23), nuclear magnetic resonance (NMR) spectroscopy (24) and dual-energy X-ray absorptiometry (24), have been used with limited success in an effort to determine features of HIV-1-infected plasma or other samples. Visible and near-infrared (Vis-NIR) spectroscopy is a fast multi-component assay which requires no reagents (25). Moreover, Vis-NIR spectroscopy is useful for identi- 
Table I. Primers used in the PCR analysis.

\begin{tabular}{lll}
\hline Usage & Name & DNA sequence \\
\hline 1st round & PRA & 5'-CCTAGGAAAAAGGGCTGTTGGAAATGTGG-3' \\
& IBR1 & 5'-AAATGATGACAGCATGTCAGGGAGT-3' \\
2nd round & PRB & 5'-ACTGAGAGACAGGCTAATTTTTTAGGGA-3' \\
& IBR2 & 5'-CAAAGGAATGGAGGTTCTTTCTGATG-3' \\
\hline
\end{tabular}

fying the chemical composition quantitatively and qualitatively when combined with chemometric analyses, such as the partial least squares regression analysis, principal component analysis (PCA), and soft independent modeling of class analogy (SIMCA) (26-28). By using Vis-NIR spectroscopy, the molecular stretching and bending combination motion of $\mathrm{X}-\mathrm{H}$, such as that of $\mathrm{O}-\mathrm{H}, \mathrm{N}-\mathrm{H}$ and $\mathrm{C}-\mathrm{H}$, can be analyzed $(25,28)$. Therefore, Vis-NIR spectroscopy has become a widely used analytical technique in the agricultural, pharmaceutical, chemical and petrochemical industries $(25,28,29)$.

Previous research has shown that it may be possible to diagnose infections of HIV-1 using plasma by a partial least squares regression analysis of Vis-NIR spectra (30). In this study, to investigate the features of HIV-infected plasma, differences in the Vis-NIR spectra of HIV-1-infected individuals and healthy donors were compared using PCA and SIMCA. A possible change in the components of plasma was explored after PCA and SIMCA of the Vis-NIR spectra.

\section{Materials and methods}

Samples. Plasma samples from 35 HIV-1-positive individuals (male/female, 32/3), reported positive at the Counseling Behavioral Modification Center (Shiraz, Iran) and confirmed by the polymerase chain reaction (PCR) method at the HIV and Hepatitis Research Center, Shiraz University of Medical Sciences (Gerash, Iran) and samples from 15 healthy volunteer donors (male/female, 15/0) were used. The HIV-1-infected individuals had not been medicated with anti-viral agents prior to blood collection. From each subject, a venous blood sample was obtained from an antecubital vein. The blood plasma was separated from blood cells by centrifugation $(2000 \mathrm{rpm}$, $10 \mathrm{~min})$. All samples were diluted 20 -fold with the addition of phosphate-buffered saline to a constant volume $(2 \mathrm{ml})$ in a polystyrene cuvette (Sarstedt, Aktiengesellschaft, Germany) before Vis-NIR spectroscopy. This research project was approved by the Ethics Committee of Osaka University and Shiraz University of Medical Sciences, and written informed consent was obtained from all of the HIV-1-infected individuals and healthy donors.

Spectra collection. Three consecutive Vis-NIR spectra were recorded for each plasma sample at a resolution of $2 \mathrm{~nm}$ with a modified portable Vis-NIR spectrophotometer (NIR Gun; Japan Fantec Research Institute, Shizuoka, Japan) at $37^{\circ} \mathrm{C}$ as described previously (26). The spectral data were measured as an absorbance value $[\log (1 / \mathrm{T})]$, where $\mathrm{T}$ is the transmittance at a wavelength ranging from 600 to $1100 \mathrm{~nm}$.

PCR. RNA was extracted with a high pure viral RNA kit (Roche Applied Science, Indianapolis, IN). First-strand cDNA was synthesized from the RNA by Moloney murine leukemia virus reverse transcriptase (Fermentase Co., Lithuania). With the cDNA, PCR for HIV-1 was performed using primers with the DNA sequences listed in Table I. PCR was first performed with the primers PRA and IBR1, then a second-round of PCR was performed with PRB and IBR2 to produce a 1200-bp product. The first PCR consisted of a hot start for $5 \mathrm{~min}$ at $94^{\circ} \mathrm{C}$, then cycles of denaturation for $20 \mathrm{sec}$ at $94^{\circ} \mathrm{C}$, annealing for $20 \mathrm{sec}$ at $52^{\circ} \mathrm{C}$, and extension for $2 \mathrm{~min}$ at $72^{\circ} \mathrm{C}$, then a final extension for $7 \mathrm{~min}$ at $72^{\circ} \mathrm{C}$. The second PCR (nested PCR) consisted of a hot start for $5 \mathrm{~min}$ at $94^{\circ} \mathrm{C}$, then cycles of denaturation for $20 \mathrm{sec}$ at $94^{\circ} \mathrm{C}$, annealing for $20 \mathrm{sec}$ at $55^{\circ} \mathrm{C}$, and extension for $1 \mathrm{~min}$ at $72^{\circ} \mathrm{C}$, then a final extension for $5 \mathrm{~min}$ at $72^{\circ} \mathrm{C}$. The detection of PCR products from HIV-1 RNA was checked by DNA electrophoresis.

Data processing. Pirouette software (version 3.11; Infometrix, Woodinville, WA) was employed for data processing. To minimize differences between spectra caused by baseline shifts and noise, prior to calibration, spectral data were mean-centered and transformed by standard normal variate (SNV) (31) and smoothing based on the Savitzky-Golay algorithm (32), and then subjected to PCA and SIMCA. The mathematical formulas of pre-processing and PCA and SIMCA are available in the Pirouette manual. For visualization of the PCA results, PCA score plots, with plot score values of two principal components (PCs), were used. The SIMCA results were visualized with a Coomans plot (33), which plots class distances against each other to assess the classification performance of the SIMCA model by predicting class membership in terms of distance from the model. The critical distance from the model used corresponded to the 0.05 level and defined a $95 \%$ tolerance interval. Through calculations of the PCA and SIMCA, loading and discriminating power were obtained.

\section{Results and Discussion}

Many observations suggest that specific changes occur in blood after HIV-1-infection, especially changes in cell population $(2,3)$. However, limited information is available regarding changes in the components of plasma after HIV-1-infection. In this study, the possible differences between the plasma compo- 


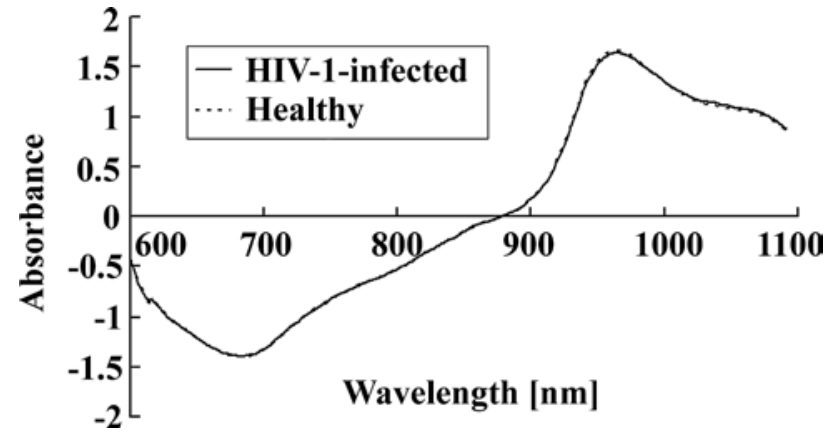

Figure 1. Visible and near-infrared (Vis-NIR) spectra of plasma from HIV-1infected and healthy individuals. A modified spectrophotometer (NIR Gun) was used to record Vis-NIR spectra from plasma at $37^{\circ} \mathrm{C}$. Transmittance spectra from HIV-1-infected and healthy individuals were collected at wavelengths of 600-1100 nm and subjected to smoothing and standard normal variates $(\mathrm{SNV})$ to minimize noise and baseline shifts. The mean spectra of the two groups are shown.

nents of HIV-1-infected individuals and healthy subjects were examined using Vis-NIR spectroscopy. To manifest these differences, chemometric analyses such as PCA and SIMCA were applied to the Vis-NIR spectra. A total of 50 subjects were examined: 35 individuals infected with HIV-1 (male/female, 33/2) and 15 healthy donors (male/female, 14/1). Both groups comprised Iranian subjects. As raw Vis-NIR spectra showed noise and a baseline shift, it was difficult to find any significant difference between HIV-1-infected and healthy individuals. Thus, to minimize noise and baseline shifts, the Vis-NIR spectra of plasma from HIV-1-infected and healthy individuals were subjected to smoothing and SNV. Several broad peaks were found in both groups in the region of 680 and $970 \mathrm{~nm}$. Very small and broad peaks were also observed at 700-800 nm, $800-900 \mathrm{~nm}$ and $1000-1100 \mathrm{~nm}$. Next, the mean spectra of the smoothed and SNV-treated data were compared between the groups. The comparison revealed very small differences in the peaks at $\sim 970 \mathrm{~nm}$ and 1000-1100 nm (Fig. 1). These results prompted us to further investigate the difference between the Vis-NIR spectra of plasma from HIV-1-infected and healthy individuals using chemometric analyses. PCA and SIMCA were applied to Vis-NIR spectra using plasma samples from the 35 HIV-1-infected and 15 healthy individuals. The results showed a relatively clear difference between plasma samples from HIV-1-infected individuals and healthy donors in terms of the PCA score plot using the first PC (PC1) versus second PC (PC2) or PC2 versus third PC (PC3) (Fig. 2). Notably, PC2 contributed to the separation of plots between HIV-1-infected and healthy individuals. Next, spectral information modeled by PCA was extracted from the corresponding loadings. PC2, which was most effective for distinguishing between the two groups, negatively peaked at $953 \mathrm{~nm}$ and positively peaked at $1038 \mathrm{~nm}$, and was slightly high at 800-900 nm and at $\sim 680 \mathrm{~nm}$. Vis-NIR spectra were subjected to SIMCA using a Coomans plot. The Coomans plot showed the reliable separation of classes of HIV-1-infected plasma from healthy plasma. The results of the PCA and SIMCA suggest that HIV-1-infections cause specific changes in plasma and affect the Vis-NIR spectra of plasma. Furthermore, as only non-medicated individuals were included in this study, drugs did not contribute to the dis-
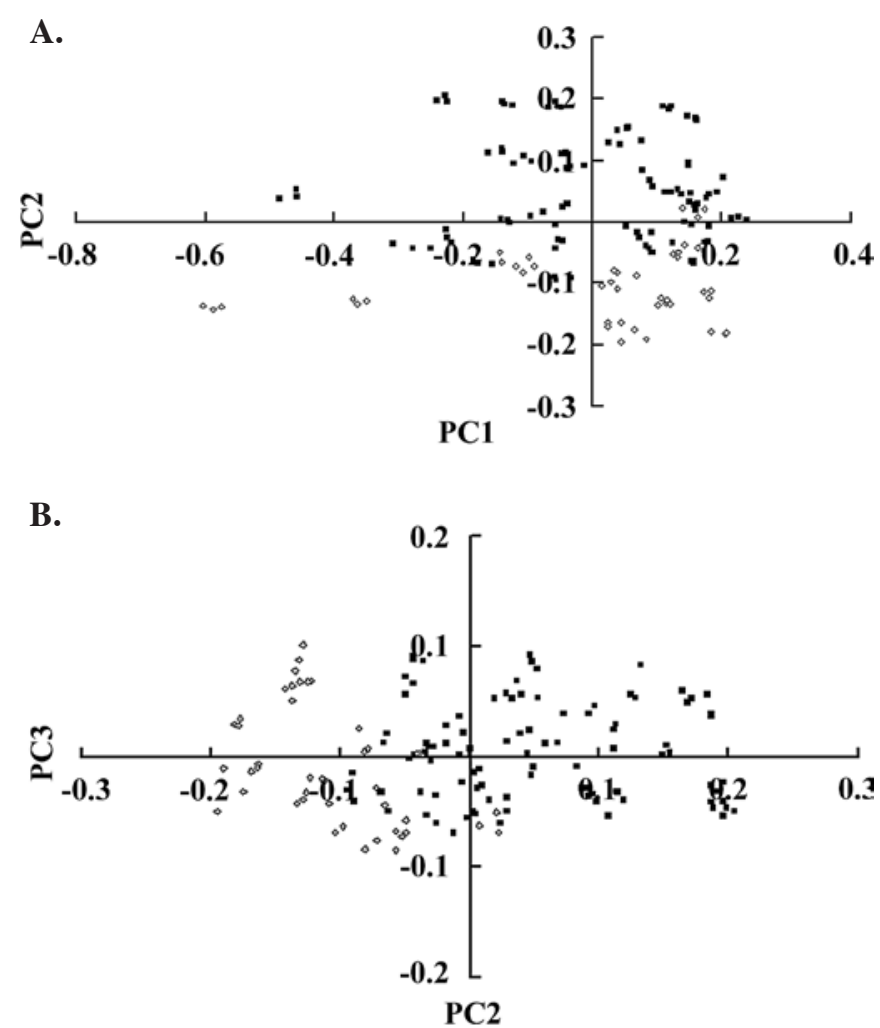

C.

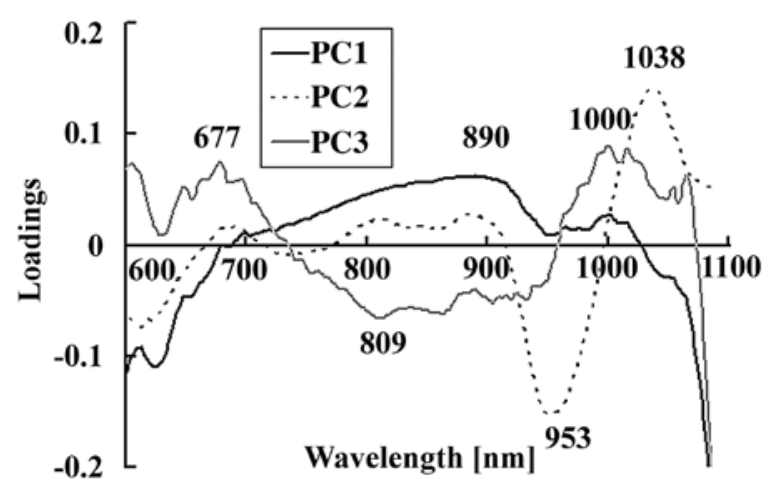

Figure 2. Principal component analysis (PCA) of visible and near-infrared (Vis-NIR) spectra from the plasma of HIV-1-infected and healthy individuals. Plasma samples from 35 HIV-1-infected and 15 healthy individuals were subjected to Vis-NIR spectroscopy. After the collection of three consecutive Vis-NIR spectra from all samples, the spectral data were pre-processed by mean centering, smoothing and standard normal variate, and were subjected to PCA. The PCA score plot of (A) the first principal component (PC1) versus the second principal component ( $\mathrm{PC} 2$ ) and (B) $\mathrm{PC} 2$ versus the third principal component (PC3) for Vis-NIR spectra from HIV-1-infected individuals (closed squares) and healthy donors (open diamonds) is shown. Samples from two individuals were hemolysed and excluded as outliers. (C) PC1, PC2 and PC3 loadings of the PCA. HIV-1-infected samples were mainly separated by PC2. The PC2 loading shows peaks at 953 and $1038 \mathrm{~nm}$, which may be assigned to the O-H stretch, 2nd overtone of ROH (alcohols) (940-970 nm) and the N-H stretch, 2nd overtone of $\mathrm{RNH}_{2}(1030 \mathrm{~nm})$, respectively.

crimination. The most prominent discriminating power, which represents the most useful wavelength in the discrimination of the two classes (HIV-1-infected and healthy individuals) in the SIMCA, had peaks at 665, 684, 859, 944 and 1027 nm (Fig. 3). The discriminating power was generally consistent with the PCA loadings, especially the PC2 loading. 

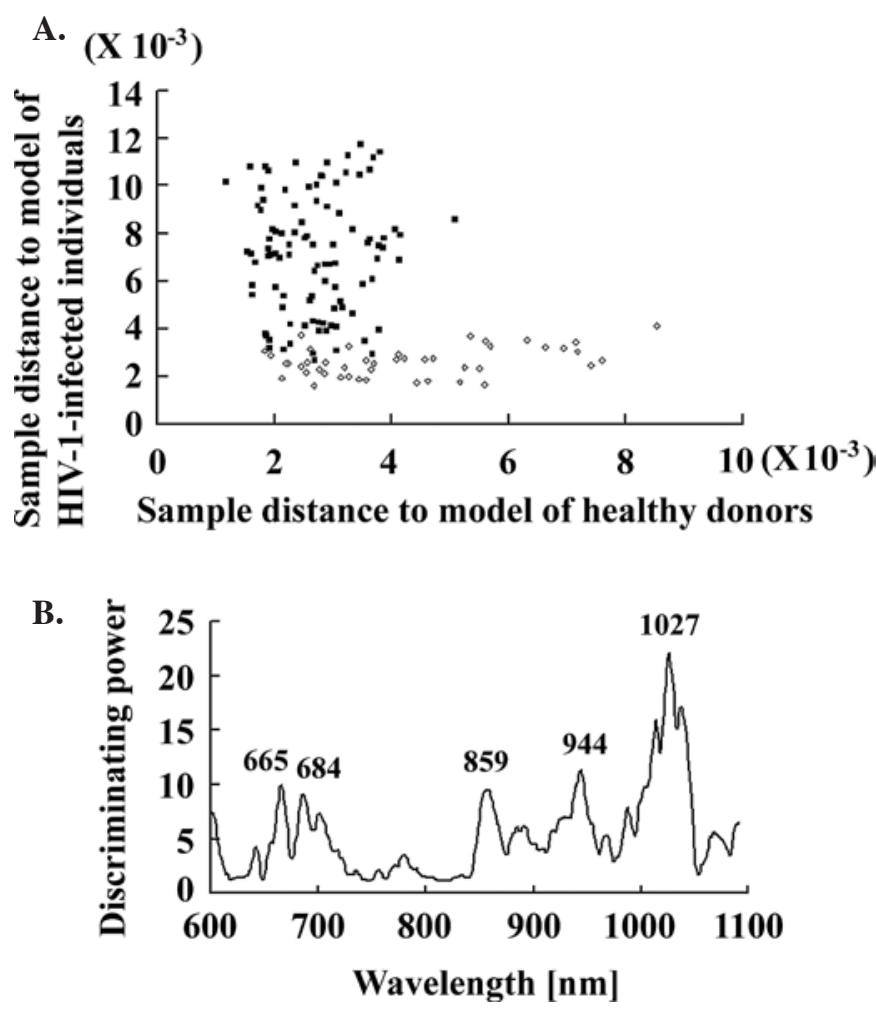

Figure 3. Soft modeling of class analogy (SIMCA) of visible and near-infrared (Vis-NIR) spectra from the plasma of HIV-1-infected and healthy individuals. Three consecutive Vis-NIR spectra of plasma from 35 HIV-1-infected and 15 healthy individuals were processed by mean centering, smoothing and standard normal variate and subjected to a SIMCA analysis. (A) A Coomans plot of the SIMCA model of HIV-1-infected individuals (closed squares) and healthy donors (open diamonds) is shown. (B) The discriminating power of the SIMCA model shows several important peaks differentiating the plasma of HIV-1-infected individuals from that of healthy donors. Samples from two individuals were hemolysed and excluded as outliers.

In this study, we examined changes in the components of plasma by analyzing Vis-NIR spectra. Plasma is composed of water, proteins (mainly albumin, fibrinogen and immunoglobulin), lipids, saccharides (mainly glucose), salts and other molecules (11). Loadings in the PCA and discriminating power in the SIMCA of Vis-NIR spectra of HIV-1-infected and healthy plasma suggest that a specific spectral pattern was found in HIV-1-infected plasma. This is because the peaks for each loading in the PCA model provide information on the absorption of plasma with which to distinguish HIV-1-infected from healthy individuals. These changes may be assigned to vibrations of $\mathrm{CH}, \mathrm{OH}$ and $\mathrm{NH}_{2}$ (Table II) (34), whose functional groups exist in proteins, lipids and saccharides. In addition, salts and metals are solubilized in water, and their concentrations may affect the vibration of water $(970 \mathrm{~nm})(35,36)$.

Recent studies using NMR, magnetic resonance imaging (MRI) and MRS have shown that components of plasma can be affected by HIV-1-infection, though information on these altered components is limited. Concerning MRI, the most prominent difference in components between HIV-1infected and healthy plasma was a decrease in the level of $N$-acetylaspartate in the brain (22). MRS also showed an increase in the ratio of the glial marker myoinositol to creatine and of choline compounds to creatine in the brain (23). NMR demonstrated increased total cholesterol and triglyceride levels in plasma and increased muscular triglyceride levels in HIV-1-infected individuals (24). Dual-energy X-ray absorptiometry indicated a decrease in the fat content in the legs of HIV-1-infected individuals and an increase in the fat content in the trunk, with a similar overall fat content as that of healthy subjects (24). Taken together, these studies suggest that HIV-1-infection disrupts neuronal integrity and causes abnormal glial activation in the brain, affecting constituents related to fats in plasma and peripheral tissues. Therefore, it would be interesting to examine whether the spectral changes observed in this study are related to the above previous studies. Further detailed analyses of Vis-NIR spectra will provide significant information on the pathophysiology of HIV-1-infection.

In conclusion, Vis-NIR analysis revealed altered constituents of HIV-1-infected plasma. This approach deserves further evaluation as a potential novel index for HIV-infection. More importantly, these results suggest the presence of factors influencing plasma by following HIV-1 infection. Further elucidation of the mechanisms causing changes in plasma may contribute to our understanding of the pathophysiology of HIV-1 infection and AIDS, and may lead to an effective treatment. Finally, we would like to emphasize that approaches to the diagnosis of HIV-1 infection through skin or the use of blood are attractive. This endeavor is now ongoing.

\section{Acknowledgements}

The authors are grateful to Dr Davarpanah, Mr. Naseri and Mr. Ansari (Counseling Behavioral Modification Center, Shiraz, Iran) and to Takanori Kobayashi (Osaka University, Osaka, Japan) for technical assistance and invaluable comments. This work was partly supported by grants-in-aid from the Japan Society for the Heiwa-Nakajima Foundation, the Kieikai Research Foundation and the Japan Science and Technology Agency.

Table II. Possible assignment of peaks in the discriminating power of the SIMCA model.

\begin{tabular}{llc}
\hline Wavelength (nm) & \multicolumn{1}{c}{ Assignment } & Refs. \\
\hline 665 & Unknown & - \\
684 & Unknown & - \\
859 & C-H stretch, 3rd overtone of ArCH (aromatics) $(857-890 \mathrm{~nm})$ & $(34)$ \\
944 & O-H stretch, 2nd overtone of $\mathrm{ROH}$ (alcohols) $(940-970 \mathrm{~nm})$ & $(34)$ \\
1027 & N-H stretch, 2nd overtone of $\mathrm{RNH}_{2}(1030 \mathrm{~nm})$ & $(34)$ \\
\hline
\end{tabular}




\section{References}

1. Ghannad MS, Arab SM, Mirzaei $M$ and Moinipur A: Epidemiologic study of human immunodeficiency virus (HIV) infection in the patients referred to health centers in Hamadan Province, Iran. AIDS Res Hum Retroviruses 25: 277-283, 2009.

2. Ikuta K, Kameoka M and Luftig RB: AIDS pathogenesis: the role of accessory gene mutations, leading to formation of longlived persistently infected cells and/or apoptosis-inducing HIV-1 particles. Virus Res 52: 145-156, 1997.

3. Perno CF, Svicher V, Schols D, Pollicita M, Balzarini J and Aquaro S: Therapeutic strategies towards HIV-1 infection in macrophages. Antiviral Res 71: 293-300, 2006.

4. Banks WA, Ercal N and Price TO: The blood-brain barrier in neuroAIDS. Curr HIV Res 4: 259-266, 2006.

5. Shor-Posner G, Basit A, Lu Y, et al: Hypocholesterolemia is associated with immune dysfunction in early human immunodeficiency virus-1 infection. Am J Med 94: 515-519, 1993.

6. Hellerstein MK, Grunfeld $\mathrm{C}, \mathrm{Wu} \mathrm{K}$, et al: Increased de novo hepatic lipogenesis in human immunodeficiency virus infection. J Clin Endocrinol Metab 76: 559-565, 1993.

7. Grunfeld C, Pang M, Doerrler W, Shigenaga JK, Jensen P and Feingold KR: Lipids, lipoproteins, triglyceride clearance, and cytokines in human immunodeficiency virus infection and the acquired immunodeficiency syndrome. J Clin Endocrinol Metab 74: 1045-1052, 1992.

8. Grunfeld C, Kotler DP, Hamadeh R, Tierney A, Wang J and Pierson RN: Hypertriglyceridemia in the acquired immunodeficiency syndrome. Am J Med 86: 27-31, 1989.

9. Constans J, Pellegrin JL, Peuchant E, et al: Plasma lipids in HIVinfected patients: a prospective study in 95 patients. Eur J Clin Invest 24: 416-420, 1994.

10. Zangerle R, Sarcletti M, Gallati H, Reibnegger G, Wachter H and Fuchs D: Decreased plasma concentrations of HDL cholesterol in HIV-infected individuals are associated with immune activation. J Acquir Immune Defic Syndr 7: 1149-1156, 1994.

11. Rose H, Hoy J, Woolley I, et al: HIV infection and high density lipoprotein metabolism. Atherosclerosis 199: 79-86, 2008.

12. Riddler SA, Smit E, Cole SR, et al: Impact of HIV infection and HAART on serum lipids in men. JAMA 289: 2978-2982, 2003.

13. Anastos K, Lu D, Shi Q, et al: Association of serum lipid levels with HIV serostatus, specific antiretroviral agents, and treatment regimens. J Acquir Immune Defic Syndr 45: 34-42, 2007.

14. Hsue PY, Lo JC, Franklin A, et al: Progression of atherosclerosis as assessed by carotid intima-media thickness in patients with HIV infection. Circulation 109: 1603-1608, 2004.

15. De Saint Martin L, Vandhuick O, Guillo P, et al: Premature atherosclerosis in HIV positive patients and cumulated time of exposure to antiretroviral therapy (SHIVA study). Atherosclerosis 185: 361-367, 2006.

16. Purnell JQ, Zambon A, Knopp RH, et al: Effect of ritonavir on lipids and post-heparin lipase activities in normal subjects. AIDS 14: 51-57, 2000.

17. Lee GA, Seneviratne T, Noor MA, et al: The metabolic effects of lopinavir/ritonavir in HIV-negative men. AIDS 18: 641-649, 2004.

18. Noor MA, Parker RA, O'Mara E, et al: The effects of HIV protease inhibitors atazanavir and lopinavir/ritonavir on insulin sensitivity in HIV-seronegative healthy adults. AIDS 18: 2137-2144, 2004
19. Alfano M, Crotti A, Vicenzi E and Poli G: New players in cytokine control of HIV infection. Curr HIV/AIDS Rep 5: 27-32, 2008.

20. Grunfeld C, Kotler DP, Shigenaga JK, et al: Circulating interferon-alpha levels and hypertriglyceridemia in the acquired immunodeficiency syndrome. Am J Med 90: 154-162, 1991.

21. Negredo E, Ribalta J, Paredes R, et al: Reversal of atherogenic lipoprotein profile in HIV-1 infected patients with lipodystrophy after replacing protease inhibitors by nevirapine. AIDS 16: 1383-1389, 2002.

22. Taylor MJ, Schweinsburg BC, Alhassoon OM, et al: Effects of human immunodeficiency virus and methamphetamine on cerebral metabolites measured with magnetic resonance spectroscopy. J Neurovirol 13: 150-159, 2007.

23. Chang L, Lee PL, Yiannoutsos CT, et al: A multicenter in vivo proton-MRS study of HIV-associated dementia and its relationship to age. Neuroimage 23: 1336-1347, 2004.

24. Luzi L, Perseghin G, Tambussi G, et al: Intramyocellular lipid accumulation and reduced whole body lipid oxidation in HIV lipodystrophy. Am J Physiol Endocrinol Metab 284: E274-E280, 2003.

25. Osborne BG and Fearn T: Near-Infrared Spectroscopy in Food Analysis. Longman Scientific \& Technical, UK, 1986.

26. Sakudo A, Kuratsune H, Kobayashi T, Tajima S, Watanabe Y and Ikuta K: Spectroscopic diagnosis of chronic fatigue syndrome by visible and near-infrared spectroscopy in serum samples. Biochem Biophys Res Commun 345: 1513-1516, 2006.

27. Sakudo A, Suganuma Y, Kobayashi T, Onodera T and Ikuta K: Near-infrared spectroscopy: promising diagnostic tool for viral infections. Biochem Biophys Res Commun 341: 279-284, 2006.

28. Ciurczak EW and Drennen JK: Pharmaceutical and Medical Applications of Near-Infrared Applications (Practical Spectroscopy). Marcel Dekker Inc., New York, NY, 2002.

29. Raghavachari R: Near-Infrared Applications in Biotechnology (Practical Spectroscopy). Marcel Dekker Inc., New York, NY, 2001.

30. Sakudo A, Tsenkova R, Onozuka T, et al: A novel diagnostic method for human immunodeficiency virus type-1 in plasma by near-infrared spectroscopy. Microbiol Immunol 49: 695-701, 2005.

31. Barnes RJ, Dhanoa MS and Lister SJ: Standard normal variate transformation and de-trending of near-infrared diffuse reflectance spectra. Appl Spectrosc 43: 772-777, 1989.

32. Savitzky A and Golay MJE: Smoothing and differentiation of data by simplified least squares procedures. Anal Chem 36: 1627-1639, 1964.

33. Coomans D, Broeckaert I, Derde MP, Tassin A, Massart DL and Wold S: Use of a microcomputer for the definition of multivariate confidence regions in medical diagnosis based on clinical laboratory profiles. Comput Biomed Res 17: 1-14, 1984.

34. Workman J: Handbook of Organic Compounds: NIR, IR, Raman and UV-Vis Spectra Featuring Polymers and Surfactants. Academic Press, San Diego, 2001.

35. Sakudo A, Yoshimura E, Tsenkova R, Ikuta K and Onodera T: Native state of metals in non-digested tissues by partial least squares regression analysis of visible and near-infrared spectra. J Toxicol Sci 32: 135-141, 2007.

36. Sakudo A, Tsenkova R, Tei K, et al: Comparison of the vibration mode of metals in $\mathrm{HNO}_{3}$ by a partial least-squares regression analysis of near-infrared spectra. Biosci Biotechnol Biochem 70: 1578-1583, 2006 